\title{
Instrumental and Sensory Analysis of the Properties of Traditional Chinese Fried Fritters
}

\author{
Daming Fan, ${ }^{1}$ Bowen Yan, ${ }^{1}$ Huizhang Lian, ${ }^{2}$ Jianxin Zhao, ${ }^{1}$ and Hao Zhang ${ }^{1}$ \\ ${ }^{1}$ State Key Laboratory of Food Science and Technology, School of Food Science and Technology, Jiangnan University, \\ Wuxi 214122, China \\ ${ }^{2}$ Wuxi Huashun Minsheng Food Co. Ltd., Wuxi 214218, China \\ Correspondence should be addressed to Hao Zhang; zhanghao@jiangnan.edu.cn
}

Received 17 July 2016; Revised 24 September 2016; Accepted 28 September 2016

Academic Editor: Yiannis Kourkoutas

Copyright (C) 2016 Daming Fan et al. This is an open access article distributed under the Creative Commons Attribution License, which permits unrestricted use, distribution, and reproduction in any medium, provided the original work is properly cited.

\begin{abstract}
The quality of traditional Chinese fried fritters is typically measured using human sensory evaluation techniques and physicochemical indices, the process of which is laborious and time-consuming. This study aimed to investigate the relationship between instrumental parameters, sensory criteria, and physicochemical indices. Significant correlations were found using principle component analysis. Volume, fat, texture, palatability, and instrumental parameters (hardness, fracturability, springiness, and gumminess) were found to be the main factors influencing the quality of Chinese fried fritters by principal component analysis (PCA) and instrumental methods, which were satisfactory replacement for human evaluation in correlation testing.
\end{abstract}

\section{Introduction}

Fried fritters are a type of traditional Chinese breakfast food and a popular snack, due to a low price and a distinctive flavor [1]. The unique taste comes from the crisp surface, loose texture, and fried flavor. China produces approximately 120 thousand tons of fritters per year, worth 2.4 billion yuan. The income of the "Yong he King" chain from the sale of fried fritters in 2003 was reported to be 300 million yuan. Classified as a traditional Chinese food, fritters are not only eaten by the Chinese but also widely consumed abroad in countries including Japan, Korea, Singapore, and Russia. However, concerns over food quality standards have become a matter of debate all over the world and have gained significant attention in China.

Since 1990, many scientific studies have focused on fried fritters. Most have centered on the development of a new formula to enhance the quality and safety [2-4]. Others have investigated the correlation between wheat flour and the quality of the final product $[5,6]$. However, the evaluation criteria have been based only on human sensory evaluation and physicochemical indices. Sensory evaluation is commonly considered to be the best qualitative method of assessment and no instrumental techniques are thought to exist to adequately replace human evaluation of food quality. However, a range of shortcomings of the human evaluation method hamper its usage, such as inconsistent evaluation standards and the relatively laborious and time-consuming process involved [7].

A significant correlation between instrument-based and subjective sensory methods has been difficult to establish and a reliable relationship is not always feasible, according to the research of Harker et al. [8]. Empirical and imitative instrumental tests have correlated with sensory texture descriptors with varying degrees of success, for fruits [7, 9-11], fried food [12, 13], bread [14], and meat products [15]. Texture profile analysis (TPA) has been used for many years as a substitute for human teeth to detect properties including hardness, fracturability, adhesiveness, springiness, cohesiveness, gumminess, chewiness, and resilience [16].

In the present study, 25 fried fritters made using different formulas were measured using both texture analysis and sensory evaluation. In order to determine the most effective instrumental parameter for the prediction of fried fritter texture, correlations between sensory perception and physicochemical and texture properties of fried fritters were investigated. 


\section{Materials and Methods}

2.1. Ingredients. Fried fritters were produced using 25 different formulas. The ingredients added for each formula were identical, and the differences between formulas were due to the amounts of the ingredients added. Flour was obtained from Tianjin (China). The leavening agent used included sodium bicarbonate (SB), gluconic acid- $\delta$-lactone $(\mathrm{GdL})$, calcium biphosphate (CB), glycerol monolaurate (GML), yeast, and amylomaize starch (AMS).

2.2. Physicochemical Properties. The moisture content of the fried fritters was determined by vacuum drying. Samples were cut into pieces (approximately $100 \mathrm{~g}$ ) and dried in a vacuum oven at $90^{\circ} \mathrm{C}$ until the weight reached a constant value (modified method 44-40 [AACC, 2000]). Lipid content was measured gravimetrically following extraction using an extraction unit (SOXTEC System HT2, 1045) with petroleum ether (boiling range $30-60^{\circ} \mathrm{C}$ ) [13]. The volumes of the samples were determined using the rapeseed displacement method [17] and five samples of each formula were selected for volume testing. Specific volume was calculated from the ratio of volume to weight. All physicochemical measurements were carried out in triplicate.

2.3. Sensory Evaluation. Nine individuals participated in the sensory evaluation test, following completion of a series of training sessions over a one-month period. Training consisted of identifying and rating the color, odor, palatability, and texture of the fried fritter samples. The criteria for each index in the sensory evaluation are listed in Table 1.

2.4. Instrumental Measurements. TPA instrumental measurements were made using AXT-2i Texture Analyzer (Stable Micro System Ltd., Surrey, UK). Seven samples were selected from each formula for TPA testing. During the testing process, samples were kept separate from any factors that could potentially influence the results, such as samples temperature and air flow. Results were averaged following removal of values outside of two standard deviations. Specific TPA parameters were as follows: probe DPH-3PB, sensitivity $10 \mathrm{~g}$, test speed $5.0 \mathrm{~mm}$ per min, speed before test $5.0 \mathrm{~mm}$ per min, speed after test $5.0 \mathrm{~mm}$ per min, test distance $70 \%$ (sample thickness), automatic trigger, and access rate 200 PPS. In instrumental measurement, many indices were obtained including hardness, fracturability, adhesiveness, springiness, cohesiveness, gumminess, chewiness, and resilience.

2.5. Statistical Analysis. Principal component analysis and correlation testing were carried out using the SPSS 17.0 software package (SPSS, Chicago, IL, USA). All instrumental and sensory data were subject to Duncan's Multiple Range Test, with the level for statistical significance set at $P<$ 0.05. Correlation coefficients were obtained using Pearson's Bivariate Correlation. Principle component analysis (PCA) was conducted to summarize the relationships between physicochemical properties and the sensory parameters.
TABLE 1: The sensory evaluation criteria of fried fritters.

\begin{tabular}{|c|c|c|}
\hline Attribute & Criteria & \\
\hline \multirow{5}{*}{ Color } & Color and brightness & 10 \\
\hline & Gold & $8 \sim 10$ \\
\hline & Light yellow or brown & $4 \sim 8$ \\
\hline & Gray or dark brown & $1 \sim 4$ \\
\hline & Aroma and taste & 10 \\
\hline \multirow{3}{*}{ Odor } & $\begin{array}{l}\text { Strong aroma of deep fried and } \\
\text { no smell }\end{array}$ & $8 \sim 10$ \\
\hline & $\begin{array}{l}\text { Strong aroma of deep fried and } \\
\text { slightly alkaline or sour taste }\end{array}$ & $4 \sim 8$ \\
\hline & $\begin{array}{l}\text { Heavier slightly alkaline or sour } \\
\text { taste }\end{array}$ & $1 \sim 4$ \\
\hline \multirow{5}{*}{ Palatability } & $\begin{array}{l}\text { The extent of tenacity and crisp } \\
\text { and chewiness }\end{array}$ & 10 \\
\hline & $\begin{array}{l}\text { Crisp and refreshing, moderately } \\
\text { tenacious, and easy to chew }\end{array}$ & $8 \sim 10$ \\
\hline & $\begin{array}{l}\text { Too soft, too hard, or difficult to } \\
\text { chew }\end{array}$ & $1 \sim 4$ \\
\hline & Middle & $4 \sim 8$ \\
\hline & Pore size and uniformity & 10 \\
\hline \multirow{3}{*}{ Texture } & Large and uniform pore & $8 \sim 10$ \\
\hline & $\begin{array}{l}\text { Large pore but general } \\
\text { uniformity }\end{array}$ & $4 \sim 8$ \\
\hline & Small pore and poor uniformity & $1 \sim 4$ \\
\hline
\end{tabular}

\section{Results and Discussion}

All results pertaining to the quality of the fried fritters are listed in Tables 2, 3, and 4. In Table 2, moisture content of all samples ranged from $27.27 \%$ to $39.67 \%$, fat content from $5.58 \%$ to $14.87 \%$, and volume from 2.22 to 3.24 . In Table 3, sample 8 had the best odor, sample 24 had the best color and texture, and sample 25 had the best palatability. In Table 4, sample 6 was best at hardness, gumminess, and chewiness and sample 3 had the best fracturability.

\subsection{Correlation between Sensory Parameters, Physicochemical} Indices, and Texture. The correlations found between sensory properties, the physicochemical indices, and TPA-derived textural measurements are displayed in Tables 5 and 6. The correlations between fat content and palatability, volume, and texture were positive and significant $(P<0.01)$. Fat content and texture, volume, and palatability were significantly and negatively correlated $(P<0.05$; Table 4$)$. This indicates that fat content and volume have direct impacts on the quality of the final product and, therefore, that the quality of fried fritters could be regulated by controlling these properties.

Fracturability and springiness were found to be significantly correlated with fat content $(P<0.05)$. The most significant correlation for fracturability, however, was with volume $(P<0.01)$. It is proposed that oil could hinder moisture absorption of the product coating as it cools, resulting in crispiness. Additionally, springiness and chewiness were found to be significantly correlated with volume. 
TABLE 2: Physicochemical results of all the fried fritters samples.

\begin{tabular}{|c|c|c|c|}
\hline Sample & Moisture content (\%) & Fat content (\%) & Volume (\%) \\
\hline 1 & $27.24 \pm 0.83^{\mathrm{b}}$ & $14.22 \pm 0.04^{\circ}$ & $2.22 \pm 0.07^{\mathrm{a}}$ \\
\hline 2 & $27.85 \pm 0.72^{b}$ & $13.62 \pm 0.04^{\mathrm{n}}$ & $2.60 \pm 0.05^{\mathrm{c}}$ \\
\hline 3 & $33.68 \pm 0.51^{\mathrm{n}}$ & $10.96 \pm 0.11^{\mathrm{j}}$ & $2.31 \pm 0.06^{\mathrm{ab}}$ \\
\hline 4 & $39.67 \pm 0.82^{\circ}$ & $9.03 \pm 0.06^{\mathrm{hi}}$ & $2.74 \pm 0.11^{\mathrm{d}}$ \\
\hline 5 & $28.95 \pm 0.61^{\mathrm{cd}}$ & $12.64 \pm 0.22^{\mathrm{m}}$ & $2.58 \pm 0.05^{\mathrm{c}}$ \\
\hline 6 & $28.33 \pm 0.29^{b c}$ & $14.87 \pm 0.29^{\mathrm{p}}$ & $2.41 \pm 0.05^{\mathrm{b}}$ \\
\hline 7 & $30.74 \pm 0.77^{\text {fgh }}$ & $12.86 \pm 0.40^{\mathrm{m}}$ & $2.40 \pm 0.07^{\mathrm{b}}$ \\
\hline 8 & $27.38 \pm 0.49^{\mathrm{b}}$ & $14.87 \pm 0.30^{\mathrm{op}}$ & $2.90 \pm 0.02^{\mathrm{def}}$ \\
\hline 9 & $32.48 \pm 0.83^{\mathrm{klm}}$ & $5.58 \pm 0.59^{\mathrm{a}}$ & $2.87 \pm 0.09^{\text {def }}$ \\
\hline 10 & $31.37 \pm 0.47^{\text {hij }}$ & $6.19 \pm 0.35^{\mathrm{cd}}$ & $2.91 \pm 0.07^{\mathrm{de}}$ \\
\hline 11 & $29.74 \pm 0.92^{\mathrm{def}}$ & $7.92 \pm 0.18^{\mathrm{f}}$ & $2.62 \pm 0.07^{c}$ \\
\hline 12 & $34.08 \pm 0.56^{\mathrm{n}}$ & $6.21 \pm 0.21^{\mathrm{bc}}$ & $3.00 \pm 0.03^{\mathrm{fg}}$ \\
\hline 13 & $30.07 \pm 0.60^{\text {efg }}$ & $6.65 \pm 0.03^{\mathrm{d}}$ & $3.00 \pm 0.03^{\mathrm{fg}}$ \\
\hline 14 & $32.55 \pm 0.38^{\mathrm{klm}}$ & $5.92 \pm 0.11^{\mathrm{ab}}$ & $2.84 \pm 0.09^{\mathrm{de}}$ \\
\hline 15 & $29.84 \pm 0.79^{\mathrm{ef}}$ & $7.29 \pm 0.24^{\mathrm{e}}$ & $3.00 \pm 0.10^{\mathrm{fg}}$ \\
\hline 16 & $31.77 \pm 0.69^{\mathrm{ijk}}$ & $8.96 \pm 0.20^{\mathrm{gh}}$ & $2.86 \pm 0.04^{\mathrm{de}}$ \\
\hline 17 & $32.36 \pm 0.87^{\mathrm{jklm}}$ & $9.32 \pm 0.11^{\mathrm{i}}$ & $3.24 \pm 0.13^{\mathrm{i}}$ \\
\hline 18 & $31.94 \pm 0.49^{\mathrm{ijk}}$ & $11.40 \pm 0.20^{\mathrm{k}}$ & $2.86 \pm 0.08^{\mathrm{de}}$ \\
\hline 19 & $32.16 \pm 0.87^{\mathrm{jkl}}$ & $11.99 \pm 0.02^{1}$ & $2.80 \pm 0.08^{\mathrm{de}}$ \\
\hline 20 & $32.99 \pm 0.64^{\operatorname{lmn}}$ & $9.39 \pm 0.20^{\mathrm{i}}$ & $3.13 \pm 0.03^{\mathrm{hi}}$ \\
\hline 21 & $30.96 \pm 0.94^{\text {ghi }}$ & $11.45 \pm 0.06^{\mathrm{k}}$ & $3.20 \pm 0.03^{\mathrm{hi}}$ \\
\hline 22 & $29.48 \pm 025^{\mathrm{de}}$ & $13.70 \pm 0.11^{\mathrm{n}}$ & $2.90 \pm 0.08^{f}$ \\
\hline 23 & $32.58 \pm 0.36^{\mathrm{klm}}$ & $7.58 \pm 0.21^{\mathrm{f}}$ & $3.07 \pm 0.04^{\mathrm{gh}}$ \\
\hline 24 & $33.39 \pm 0.41^{\mathrm{mn}}$ & $6.95 \pm 0.25^{\mathrm{d}}$ & $3.24 \pm 0.13^{\mathrm{i}}$ \\
\hline 25 & $33.42 \pm 0.59^{\mathrm{mn}}$ & $8.77 \pm 0.44^{\mathrm{g}}$ & $3.21 \pm 0.06^{\mathrm{i}}$ \\
\hline
\end{tabular}

Results as mean \pm SD. Different superscripts in the same row denote significant differences $(P<0.05)$.

Textural properties also showed good correlation with sensory parameters, as shown in Table 6. A significant correlation was found between texture and fracturability, gumminess, and chewiness $(P<0.05)$. Hardness, fracturability, and chewiness were closely associated with palatability $(P<$ $0.05)$. This indicates that the TPA technique could feasibly take the place of human sensory evaluation for fried fritters.

3.2. Principal Component Analysis. Fifteen indices including sensory evaluation and physicochemical and textural properties were analyzed by PCA. Four main components were selected according to their eigenvalue. Eigenvalues and cumulative percentages are listed in Table 7 . The loads of each index on the main components are listed in Table 8.

The PCA results demonstrated that the first four principal components (PC1, PC2, PC3, and PC4) accounted for 33.05\%, $18.12 \%, 12.56 \%$, and $11.20 \%$ of the total variance, respectively, with a combined total of $74.93 \%$ (Table 7 ).

The variables relating to the quality of fried fritters were significantly explained by the indices represented by PC1, since $\mathrm{PCl}$ explained most of the total variation (Figure 1). The correlation between the indices distributed on different axes was found to be poor. The correlation between every two indices distributed on two ends of one axis was found to be negative. PCl was mainly influenced by chewiness,

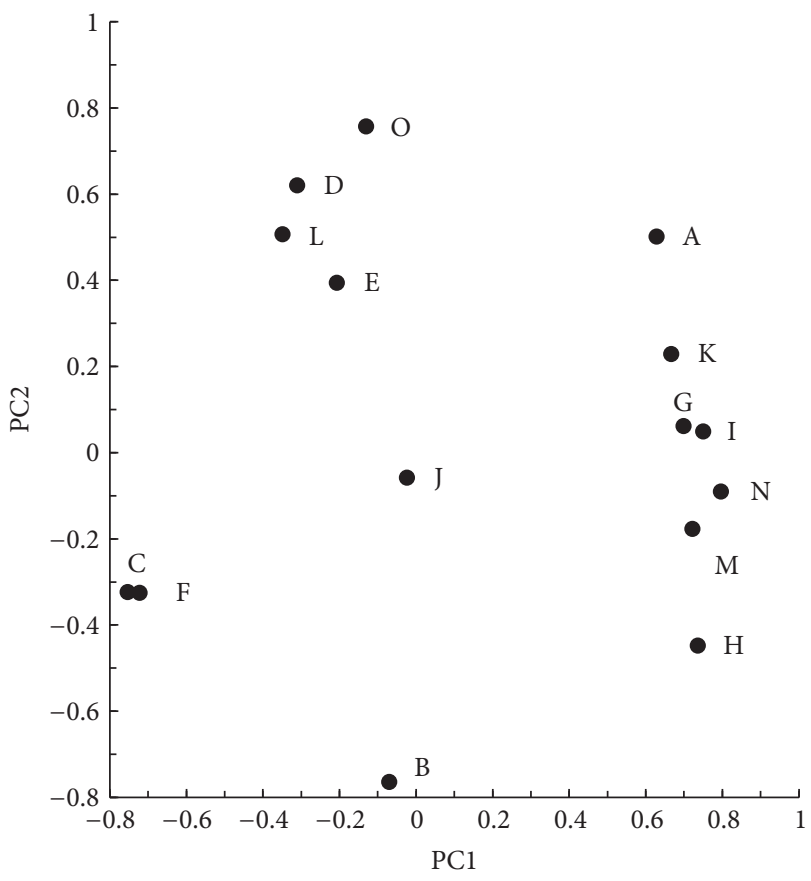

FIGURE 1: The loading plot of term of fried fritters in 1 and 2 principal components (PC1 and PC2).

fracturability, palatability, hardness, springiness, gumminess, moisture, volume, and texture (Table 8, Figure 1). Volume and texture were found to be negatively correlated with chewiness, fracturability, hardness, palatability, moisture, springiness, and gumminess. Volume and texture were along the negative axis, while chewiness, fracturability, hardness, palatability, moisture, springiness, and gumminess were along the positive axis (Figure 1).

The main factors for PC2 were resilience, odor, and fat content. However, resilience and odor were found to be negatively correlated with fat content. PC3 was mainly influenced by color and cohesiveness (Table 8, Figure 2) and they were distributed on the positive and negative dimension of axis, respectively. Moisture, palatability, and gumminess were the main variables explained by PC4. Moisture and palatability were loaded on the positive dimension while gumminess was on the negative dimension.

From Table 9 and Figure 3, the nature differences of samples are determined by the relative distance from the loading plots. The results for samples 3, 4, and 6 indicate that they rated highly for chewiness, frangibility, hardness, palatability, moisture, springiness, and gumminess, and samples $13,11,15$, 21,17 , and 24 rated highly for volume and texture (Table 9 , Figure 3). The results for sample 9 indicate that it rated highly for resilience and odor. Samples 6 and 4 not only rated highly for texture properties but also had high fat content (Figure 3).

As shown in Table 9 and Figure 4, the color of samples 8, 25 , and 24 was much better than the others. Samples 5, 14, 13, and 7 were found to have the best cohesiveness. Sample 7 had not only high cohesiveness but also the highest water content. Samples 23 and 24 also had high moisture and a pleasing color. Samples 6 and 21 rated highly for gumminess. 
TABLE 3: Sensory results of all the fried fritters samples.

\begin{tabular}{|c|c|c|c|c|}
\hline Sample & Odor & Color & Texture & Palatability \\
\hline 1 & $7.56 \pm 0.88^{\mathrm{ab}}$ & $7.00 \pm 1.50^{\mathrm{a}}$ & $4.44 \pm 1.24^{\mathrm{a}}$ & $5.00 \pm 0.83^{\mathrm{a}}$ \\
\hline 2 & $5.78 \pm 1.09^{\mathrm{ab}}$ & $6.67 \pm 0.87^{\mathrm{a}}$ & $5.78 \pm 1.72^{\mathrm{ab}}$ & $5.67 \pm 0.71^{\mathrm{ab}}$ \\
\hline 3 & $5.67 \pm 1.22^{\mathrm{ab}}$ & $7.00 \pm 1.12^{\mathrm{a}}$ & $5.00 \pm 1.32^{\mathrm{ab}}$ & $5.67 \pm 1.00^{\mathrm{ab}}$ \\
\hline 4 & $5.44 \pm 1.24^{\mathrm{ab}}$ & $5.78 \pm 1.48^{\mathrm{a}}$ & $5.89 \pm 1.90^{\mathrm{ab}}$ & $5.56 \pm 0.93^{\mathrm{ab}}$ \\
\hline 5 & $5.00 \pm 1.14^{\mathrm{a}}$ & $5.67 \pm 1.66^{\mathrm{a}}$ & $5.56 \pm 1.13^{\mathrm{ab}}$ & $5.56 \pm 1.41^{\mathrm{ab}}$ \\
\hline 6 & $5.56 \pm 1.24^{\mathrm{ab}}$ & $5.78 \pm 2.39^{\mathrm{a}}$ & $5.33 \pm 1.32^{\mathrm{ab}}$ & $6.11 \pm 1.05^{\mathrm{ab}}$ \\
\hline 7 & $5.22 \pm 1.3^{\mathrm{ab}}$ & $4.67 \pm 3.28^{\mathrm{a}}$ & $5.22 \pm 1.30^{\mathrm{ab}}$ & $6.33 \pm 1.13^{\mathrm{ab}}$ \\
\hline 8 & $7.67 \pm 1.22^{\mathrm{b}}$ & $7.33 \pm 1.41^{\mathrm{a}}$ & $7.11 \pm 0.78^{\mathrm{ab}}$ & $5.89 \pm 0.50^{\mathrm{ab}}$ \\
\hline 9 & $7.00 \pm 0.87^{\mathrm{ab}}$ & $7.00 \pm 0.87^{\mathrm{a}}$ & $6.56 \pm 1.81^{\mathrm{ab}}$ & $6.22 \pm 0.88^{\mathrm{ab}}$ \\
\hline 10 & $5.78 \pm 1.48^{\mathrm{ab}}$ & $6.11 \pm 1.27^{\mathrm{a}}$ & $7.11 \pm 1.69^{\mathrm{ab}}$ & $7.22 \pm 1.74^{\mathrm{ab}}$ \\
\hline 11 & $7.11 \pm 1.27^{\mathrm{ab}}$ & $6.89 \pm 0.78^{\mathrm{a}}$ & $5.78 \pm 1.64^{\mathrm{ab}}$ & $7.22 \pm 0.93^{\mathrm{ab}}$ \\
\hline 12 & $6.22 \pm 1.3^{\mathrm{ab}}$ & $5.89 \pm 1.05^{\mathrm{a}}$ & $7.44 \pm 2.24^{\mathrm{ab}}$ & $7.44 \pm 1.20^{\mathrm{ab}}$ \\
\hline 13 & $5.78 \pm 1.86^{\mathrm{ab}}$ & $5.22 \pm 1.92^{\mathrm{a}}$ & $7.11 \pm 1.62^{\mathrm{ab}}$ & $6.67 \pm 2.65^{\mathrm{ab}}$ \\
\hline 14 & $5.44 \pm 1.42^{\mathrm{ab}}$ & $4.72 \pm 3.63^{\mathrm{a}}$ & $6.00 \pm 1.22^{\mathrm{ab}}$ & $6.78 \pm 1.80^{\mathrm{ab}}$ \\
\hline 15 & $7.33 \pm 1.22^{\mathrm{ab}}$ & $7.67 \pm 0.71^{\mathrm{a}}$ & $7.33 \pm 1.50^{\mathrm{ab}}$ & $6.89 \pm 2.29^{\mathrm{ab}}$ \\
\hline 16 & $5.44 \pm 1.59^{\mathrm{ab}}$ & $5.56 \pm 1.74^{\mathrm{a}}$ & $6.78 \pm 1.39^{\mathrm{ab}}$ & $7.00 \pm 1.87^{\mathrm{ab}}$ \\
\hline 17 & $7.22 \pm 1.3^{\mathrm{ab}}$ & $7.78 \pm 0.76^{\mathrm{a}}$ & $7.67 \pm 1.32^{b}$ & $7.44 \pm 1.12^{\mathrm{b}}$ \\
\hline 18 & $7.33 \pm 1.22^{\mathrm{ab}}$ & $7.33 \pm 2.35^{\mathrm{a}}$ & $6.33 \pm 1.22^{\mathrm{ab}}$ & $6.67 \pm 1.39^{\mathrm{ab}}$ \\
\hline 19 & $6.44 \pm 1.01^{\mathrm{ab}}$ & $7.22 \pm 0.83^{\mathrm{a}}$ & $6.11 \pm 1.54^{\mathrm{ab}}$ & $7.00 \pm 1.39^{\mathrm{ab}}$ \\
\hline 20 & $5.78 \pm 1.99^{\mathrm{ab}}$ & $6.22 \pm 1.92^{\mathrm{a}}$ & $7.56 \pm 2.19^{\mathrm{b}}$ & $7.11 \pm 0.97^{\mathrm{ab}}$ \\
\hline 21 & $7 \pm 1.32^{\mathrm{ab}}$ & $7.22 \pm 1.09^{\mathrm{a}}$ & $7.67 \pm 1.66^{\mathrm{b}}$ & $7.33 \pm 1.45^{\mathrm{ab}}$ \\
\hline 22 & $6.89 \pm 1.27^{\mathrm{ab}}$ & $6.56 \pm 1.24^{\mathrm{a}}$ & $6.89 \pm 1.17^{\mathrm{ab}}$ & $7.78 \pm 2.35^{\mathrm{ab}}$ \\
\hline 23 & $5.56 \pm 0.88^{\mathrm{ab}}$ & $5.78 \pm 1.20^{\mathrm{a}}$ & $7.56 \pm 1.74^{\mathrm{b}}$ & $7.89 \pm 1.05^{\mathrm{b}}$ \\
\hline 24 & $6.22 \pm 0.97^{\mathrm{ab}}$ & $7.78 \pm 0.67^{\mathrm{a}}$ & $7.89 \pm 0.93^{b}$ & $7.67 \pm 1.22^{\mathrm{ab}}$ \\
\hline 25 & $6.44 \pm 1.42^{\mathrm{ab}}$ & $7.67 \pm 2.12^{\mathrm{a}}$ & $7.78 \pm 1.30^{\mathrm{b}}$ & $8.11 \pm 1.27^{\mathrm{b}}$ \\
\hline
\end{tabular}

Results as mean \pm SD. Different superscripts in the same row denote significant differences $(P<0.05)$.

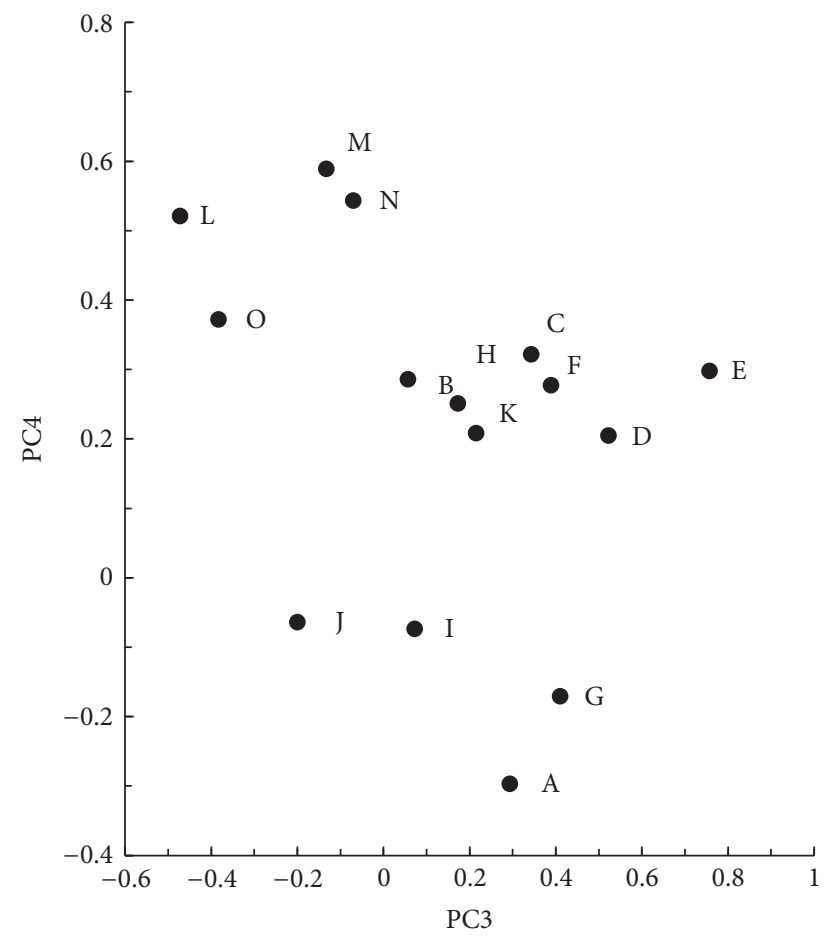

FIgURE 2: The loading plot of term of fried fritters in 3 and 4 principal components (PC3 and PC4).

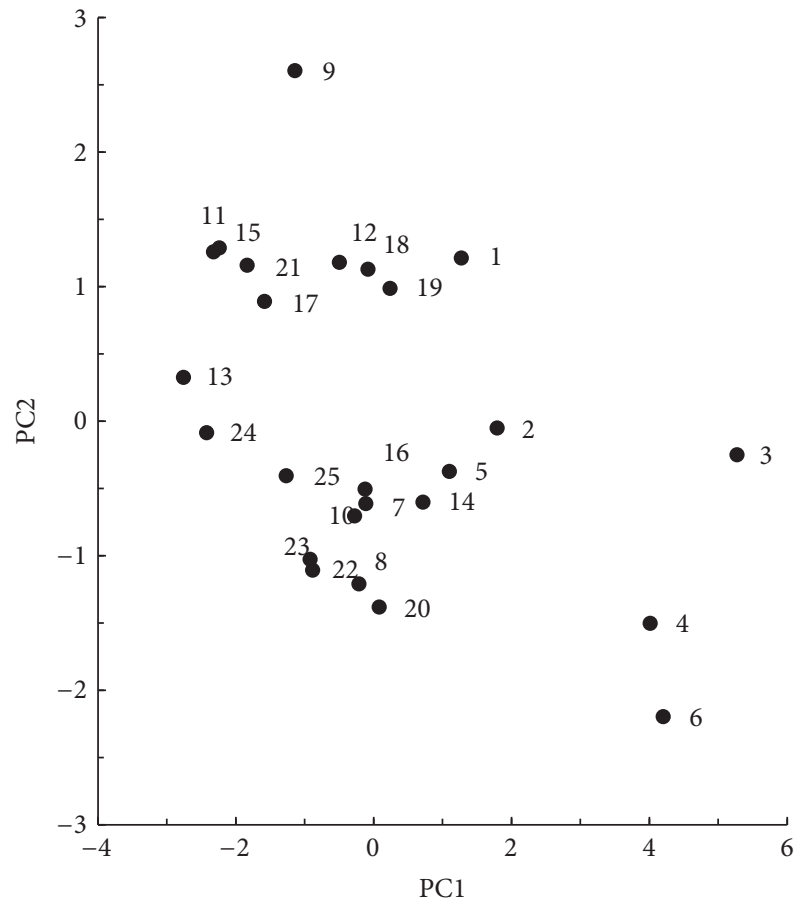

FIgURE 3: The score plot of samples in 1 and 2 principal components (PC1 and PC2). 
TABLE 4: Texture properties of all the fried fritters samples.

\begin{tabular}{|c|c|c|c|c|c|c|c|c|}
\hline Sample & Hardness (g) & Fracturability & Adhesiveness & Springiness & Cohesiveness & Gumminess & Chewiness & Resilience \\
\hline 1 & $1941^{\text {bcde }}$ & $29.26^{b c}$ & $0.19^{\mathrm{ab}}$ & $0.87^{\mathrm{abc}}$ & $0.48^{\mathrm{a}}$ & $1005^{\mathrm{abcd}}$ & $874^{\text {abcd }}$ & $0.15^{\mathrm{abcd}}$ \\
\hline 2 & $2036^{\mathrm{abcd}}$ & $46.36^{\mathrm{de}}$ & $-0.52^{\mathrm{a}}$ & $0.87^{\mathrm{abc}}$ & $0.51^{\mathrm{abcd}}$ & $1039^{\mathrm{abcd}}$ & $903^{\mathrm{abcd}}$ & $0.16^{\mathrm{abcd}}$ \\
\hline 3 & $2413^{\mathrm{de}}$ & $51.59^{\mathrm{e}}$ & $0.74^{\mathrm{ab}}$ & $0.93^{\mathrm{c}}$ & $0.47^{\mathrm{abcd}}$ & $1141^{\mathrm{bcd}}$ & $1061^{\mathrm{cd}}$ & $0.14^{\mathrm{ab}}$ \\
\hline 4 & $2667^{e}$ & $36.93^{\mathrm{cd}}$ & $0.15^{\mathrm{ab}}$ & $0.78^{\mathrm{a}}$ & $0.45^{\mathrm{ab}}$ & $1194^{\mathrm{cd}}$ & $932^{\mathrm{bcd}}$ & $0.13^{\mathrm{a}}$ \\
\hline 5 & $2015^{\mathrm{abcd}}$ & $21.85^{\mathrm{ab}}$ & $-0.57^{\mathrm{a}}$ & $0.82^{\mathrm{ab}}$ & $0.51^{\mathrm{abcd}}$ & $1055^{\mathrm{abcd}}$ & $871^{\text {abcd }}$ & $0.17^{\mathrm{cd}}$ \\
\hline 6 & $2696^{\mathrm{e}}$ & $17.09^{\mathrm{ab}}$ & $0.65^{\mathrm{ab}}$ & $0.90^{b c}$ & $0.47^{\mathrm{abcd}}$ & $1282^{\mathrm{d}}$ & $1157^{\mathrm{d}}$ & $0.15^{\mathrm{abcd}}$ \\
\hline 7 & $1571^{\mathrm{a}}$ & $16.56^{\mathrm{ab}}$ & $0.18^{\mathrm{ab}}$ & $0.78^{\mathrm{a}}$ & $0.49^{\mathrm{abcd}}$ & $778^{\mathrm{abc}}$ & $606^{\mathrm{ab}}$ & $0.15^{\mathrm{abcd}}$ \\
\hline 8 & $2004^{\mathrm{abcd}}$ & $15.37^{\mathrm{ab}}$ & $0.08^{\mathrm{ab}}$ & $0.87^{\mathrm{abc}}$ & $0.45^{\mathrm{ab}}$ & $904^{\mathrm{abcd}}$ & $787^{\mathrm{abcd}}$ & $0.14^{\mathrm{abcd}}$ \\
\hline 9 & $1808^{\mathrm{abc}}$ & $10.42^{\mathrm{a}}$ & $0.02^{\mathrm{ab}}$ & $0.82^{\mathrm{abc}}$ & $0.55^{\mathrm{d}}$ & $984^{\mathrm{abcd}}$ & $807^{\mathrm{abcd}}$ & $0.17^{\mathrm{bcd}}$ \\
\hline 10 & $2127^{\mathrm{bcd}}$ & $10.20^{\mathrm{a}}$ & $0.03^{\mathrm{ab}}$ & $0.80^{\mathrm{ab}}$ & $0.50^{\mathrm{abcd}}$ & $1068^{\mathrm{abcd}}$ & $862^{\mathrm{abcd}}$ & $0.14^{\mathrm{abcd}}$ \\
\hline 11 & $1748^{\mathrm{ab}}$ & $9.97^{\mathrm{a}}$ & $0.10^{\mathrm{ab}}$ & $0.79^{\mathrm{ab}}$ & $0.51^{\mathrm{abcd}}$ & $638^{\mathrm{a}}$ & $514^{\mathrm{a}}$ & $0.15^{\mathrm{abcd}}$ \\
\hline 12 & $1875^{\mathrm{ab}}$ & $11.36^{\mathrm{a}}$ & $-0.44^{\mathrm{a}}$ & $0.84^{\mathrm{abc}}$ & $0.53^{\mathrm{cd}}$ & $1001^{\mathrm{abcd}}$ & $837^{\mathrm{abcd}}$ & $0.16^{\mathrm{abcd}}$ \\
\hline 13 & $1525^{\mathrm{a}}$ & $12.93^{\mathrm{a}}$ & $1.68^{\mathrm{b}}$ & $0.80^{\mathrm{ab}}$ & $0.51^{\mathrm{abcd}}$ & $781^{\mathrm{abc}}$ & $628^{\mathrm{ab}}$ & $0.16^{\mathrm{abcd}}$ \\
\hline 14 & $2193^{b c d}$ & $10.16^{\mathrm{a}}$ & $0.48^{\mathrm{ab}}$ & $0.82^{\mathrm{abc}}$ & $0.50^{\mathrm{abcd}}$ & $1088^{\mathrm{abcd}}$ & $896^{\mathrm{abcd}}$ & $0.15^{\mathrm{abcd}}$ \\
\hline 15 & $1795^{\mathrm{abc}}$ & $10.92^{\mathrm{a}}$ & $0.35^{\mathrm{ab}}$ & $0.80^{\mathrm{ab}}$ & $0.51^{\mathrm{abcd}}$ & $914^{\mathrm{abcd}}$ & $733^{\mathrm{abc}}$ & $0.16^{\mathrm{abcd}}$ \\
\hline 16 & $1912^{\mathrm{abcd}}$ & $11.54^{\mathrm{a}}$ & $-0.29^{\mathrm{ab}}$ & $0.82^{\mathrm{ab}}$ & $0.48^{\mathrm{abcd}}$ & $902^{\mathrm{abcd}}$ & $737^{\mathrm{abc}}$ & $0.15^{\mathrm{abcd}}$ \\
\hline 17 & $1890^{\mathrm{abc}}$ & $12.13^{\mathrm{a}}$ & $0.08^{\mathrm{ab}}$ & $0.81^{\mathrm{ab}}$ & $0.51^{\mathrm{abcd}}$ & $968^{\mathrm{abcd}}$ & $785^{\mathrm{abcd}}$ & $0.16^{\mathrm{abcd}}$ \\
\hline 18 & $1950^{\mathrm{abcd}}$ & $11.12^{\mathrm{a}}$ & $0.54^{\mathrm{ab}}$ & $0.83^{\mathrm{abc}}$ & $0.52^{\text {bcd }}$ & $1009^{\mathrm{abcd}}$ & $834^{\mathrm{abcd}}$ & $0.16^{\mathrm{abcd}}$ \\
\hline 19 & $2271^{\text {cde }}$ & $13.02^{\mathrm{a}}$ & $0.04^{\mathrm{ab}}$ & $0.81^{\mathrm{ab}}$ & $0.54^{\mathrm{cd}}$ & $965^{\mathrm{abcd}}$ & $761^{\mathrm{abcd}}$ & $0.17^{\mathrm{abcd}}$ \\
\hline 20 & $2156^{\mathrm{bcd}}$ & $10.54^{\mathrm{a}}$ & $-0.15^{\mathrm{ab}}$ & $0.83^{\mathrm{abc}}$ & $0.47^{\mathrm{abcd}}$ & $1012^{\mathrm{abcd}}$ & $839^{\mathrm{abcd}}$ & $0.14^{\mathrm{abc}}$ \\
\hline 21 & $1728^{\mathrm{ab}}$ & $10.46^{\mathrm{a}}$ & $0.19^{\mathrm{ab}}$ & $0.83^{\mathrm{abc}}$ & $0.54^{\mathrm{d}}$ & $935^{\text {abcd }}$ & $775^{\mathrm{abcd}}$ & $0.17^{\mathrm{d}}$ \\
\hline 22 & $1851^{\mathrm{abc}}$ & $18.19^{\mathrm{ab}}$ & $0.59^{\mathrm{ab}}$ & $0.79^{\mathrm{ab}}$ & $0.48^{\mathrm{abcd}}$ & $888^{\mathrm{abcd}}$ & $701^{a b c}$ & $0.14^{\mathrm{abc}}$ \\
\hline 23 & $2168^{\mathrm{bcd}}$ & $9.88^{\mathrm{a}}$ & $-0.21^{\mathrm{ab}}$ & $0.84^{\mathrm{abc}}$ & $0.46^{\mathrm{abc}}$ & $706^{\mathrm{ab}}$ & $582^{\mathrm{ab}}$ & $0.14^{\mathrm{ab}}$ \\
\hline 24 & $1938^{\mathrm{abcd}}$ & $12.39^{\mathrm{a}}$ & $0.04^{\mathrm{ab}}$ & $0.78^{\mathrm{a}}$ & $0.47^{\mathrm{abcd}}$ & $680^{\mathrm{a}}$ & $539^{\mathrm{ab}}$ & $0.14^{\mathrm{abcd}}$ \\
\hline 25 & $1858^{\mathrm{ab}}$ & $10.33^{\mathrm{a}}$ & $-0.07^{\mathrm{ab}}$ & $0.84^{\mathrm{abc}}$ & $0.46^{\mathrm{abc}}$ & $862^{\mathrm{abcd}}$ & $726^{\mathrm{abc}}$ & $0.14^{\mathrm{abcd}}$ \\
\hline
\end{tabular}

Different superscripts in the same row denote significant differences $(P<0.05)$.

TABLE 5: The correlation between sensory evaluation and physicochemical indexes.

\begin{tabular}{lccccc}
\hline & & Odor & Color & Texture & Palatability \\
\hline \multirow{2}{*}{ Moisture } & Correlation & -0.301 & -0.076 & 0.251 & 0.243 \\
& Sig & 0.143 & 0.717 & 0.227 & 0.243 \\
Fat & Correlation & 0.125 & 0.121 & $-0.493^{*}$ & $0.799^{* *}$ \\
& Sig & 0.551 & 0.565 & 0.012 & 0.000 \\
\multirow{2}{*}{ Volume } & Correlation & 0.210 & 0.299 & $0.972^{* *}$ & $-0.442^{*}$ \\
& Sig & 0.315 & 0.146 & 0.000 & 0.027 \\
\hline
\end{tabular}

* indicates statistical significance $P<0.05$ and $* *$ indicates statistical significance $P<0.01$.

TABLE 6: The correlation between sensory evaluation and TPA results.

\begin{tabular}{lcccccccc}
\hline & Hardness & Fracturability & Adhesiveness & Springiness & Cohesiveness & Gumminess & Chewiness & Resilience \\
\hline Odor & $-0.340^{*}$ & -0.170 & 0.026 & 0.040 & 0.264 & -0.262 & -0.223 & 0.310 \\
Color & -0.119 & 0.009 & -0.120 & 0.124 & 0.082 & -0.173 & -0.119 & 0.134 \\
Texture & -0.320 & $-0.601^{* *}$ & -0.055 & -0.343 & -0.032 & 0.340 & $-0.397^{*}$ & -0.193 \\
Palatability & $0.552^{* *}$ & $0.478^{* *}$ & -0.141 & 0.376 & -0.339 & 0.389 & $0.415^{*}$ & -0.174 \\
\hline
\end{tabular}

* indicates statistical significance $P<0.05$ and ** indicates statistical significance $P<0.01$.

\section{Conclusions}

The instrumental data demonstrated high correlations between physicochemical properties and sensory and textural properties of the Chinese traditional fired fritters.
Meanwhile, principle component analysis revealed that volume, fat, texture, palatability, hardness, fracturability, springiness, and gumminess were the main factors to influence their inherent qualities. To date, a novel way is needed to improve the quality of evaluation system, and 
TABLE 7: Eigenvalues and cumulative percentage of first five principal components.

\begin{tabular}{lccc}
\hline Principle components & Eigenvalues & Initial percentage, $\%$ & Cumulative percentage, $\%$ \\
\hline PC1 & 4.958 & 33.050 & 33.050 \\
PC2 & 2.718 & 18.121 & 51.172 \\
PC3 & 1.884 & 12.562 & 63.733 \\
PC4 & 1.678 & 11.189 & 74.922 \\
\hline
\end{tabular}

TABLE 8: Eigenvalues for principal components for all the parameters of fried fritters.

\begin{tabular}{|c|c|c|c|c|c|}
\hline Mark & Properties & $\mathrm{PC1}$ & PC2 & PC3 & PC4 \\
\hline A & Moisture & 0.628 & 0.501 & 0.293 & -0.297 \\
\hline B & Fat & -0.070 & -0.764 & 0.057 & 0.286 \\
\hline $\mathrm{C}$ & Volume & -0.754 & -0.324 & 0.343 & 0.322 \\
\hline $\mathrm{D}$ & Odor & -0.311 & 0.620 & 0.522 & 0.205 \\
\hline $\mathrm{E}$ & Color & -0.207 & 0.394 & 0.757 & 0.298 \\
\hline $\mathrm{F}$ & Texture & -0.722 & -0.325 & 0.389 & 0.277 \\
\hline G & Palatability & 0.699 & 0.062 & 0.410 & -0.171 \\
\hline $\mathrm{H}$ & Hardness & 0.736 & -0.448 & 0.173 & 0.251 \\
\hline I & Fracturability & 0.750 & 0.049 & 0.072 & -0.074 \\
\hline $\mathrm{J}$ & Adhesiveness & -0.024 & -0.058 & -0.200 & -0.064 \\
\hline K & Springiness & 0.666 & 0.229 & 0.215 & 0.208 \\
\hline $\mathrm{L}$ & Cohesiveness & -0.349 & 0.507 & -0.472 & 0.521 \\
\hline M & Gumminess & 0.722 & -0.177 & -0.133 & 0.589 \\
\hline $\mathrm{N}$ & Chewiness & 0.796 & -0.090 & -0.070 & 0.543 \\
\hline $\mathrm{O}$ & Resilience & -0.131 & 0.757 & -0.383 & 0.372 \\
\hline
\end{tabular}

TABLE 9: Eigenvalues of samples in 1,2,3, and 4 principal components.

\begin{tabular}{|c|c|c|c|c|c|c|}
\hline Sample & $F 1$ & $F 2$ & $F 3$ & $F 4$ & $F$ & Ranking \\
\hline 1 & 1.2695 & 1.2107 & -0.1603 & 0.1716 & 0.7327 & 4 \\
\hline 2 & 1.7937 & -0.0523 & -0.4295 & 1.3033 & 0.7116 & 5 \\
\hline 3 & 5.2734 & -0.2503 & 0.5776 & 0.1368 & 2.3217 & 1 \\
\hline 4 & 4.013 & -1.5026 & 1.0813 & -1.3563 & 1.2287 & 3 \\
\hline 5 & 1.101 & -0.3736 & -2.0916 & 0.6963 & -0.0528 & 12 \\
\hline 6 & 4.2018 & -2.1955 & -0.7042 & 1.8758 & 1.4382 & 2 \\
\hline 7 & -0.1128 & -0.6137 & -2.2215 & -2.7943 & -0.997 & 24 \\
\hline 8 & -0.2136 & -1.2094 & 2.2814 & 0.3512 & 0.0864 & 9 \\
\hline 9 & -1.1416 & -2.6044 & -0.9615 & 1.2528 & 0.1426 & 8 \\
\hline 10 & -0.2772 & -0.7054 & -0.7454 & 0.0212 & -0.3469 & 17 \\
\hline 11 & -2.3214 & 1.2577 & -0.4492 & -1.8035 & -0.9713 & 23 \\
\hline 12 & -0.4964 & 1.1785 & -0.3499 & 0.8183 & 0.0372 & 10 \\
\hline 13 & -2.7597 & 0.3237 & -2.4175 & -1.0632 & -1.2855 & 25 \\
\hline 14 & 0.7143 & -0.6014 & -2.3617 & -0.1547 & -0.1429 & 14 \\
\hline 15 & -2.2415 & 1.2856 & 0.3539 & 0.8113 & -0.3732 & 18 \\
\hline 16 & -0.124 & -0.5061 & -0.6572 & -1.0559 & -0.487 & 19 \\
\hline 17 & -1.5841 & 0.8898 & 1.4251 & 1.3191 & -0.0469 & 11 \\
\hline 18 & -0.0822 & 1.1271 & 0.4473 & 1.1259 & 0.4837 & 6 \\
\hline 19 & 0.2398 & 0.9856 & -0.1132 & 1.1376 & 0.3365 & 7 \\
\hline 20 & 0.0801 & -1.3817 & 1.042 & 0.0356 & -0.1356 & 13 \\
\hline 21 & -1.8335 & 1.1578 & 0.3848 & 1.8044 & -0.2052 & 15 \\
\hline 22 & -0.8832 & -1.1076 & 0.742 & -0.655 & -0.5288 & 20 \\
\hline 23 & -0.9238 & -1.0279 & 1.0723 & -1.957 & -0.7463 & 21 \\
\hline 24 & -2.4238 & -0.0873 & 2.2192 & -1.6996 & -0.8961 & 22 \\
\hline 25 & -1.2677 & -0.4059 & 2.0356 & -0.5125 & -0.3297 & 16 \\
\hline
\end{tabular}

$F 1, F 2, F 3, F 4$, and $F$ stand for eigenvalues of the first, second, third, fourth, and total principal component, respectively. 


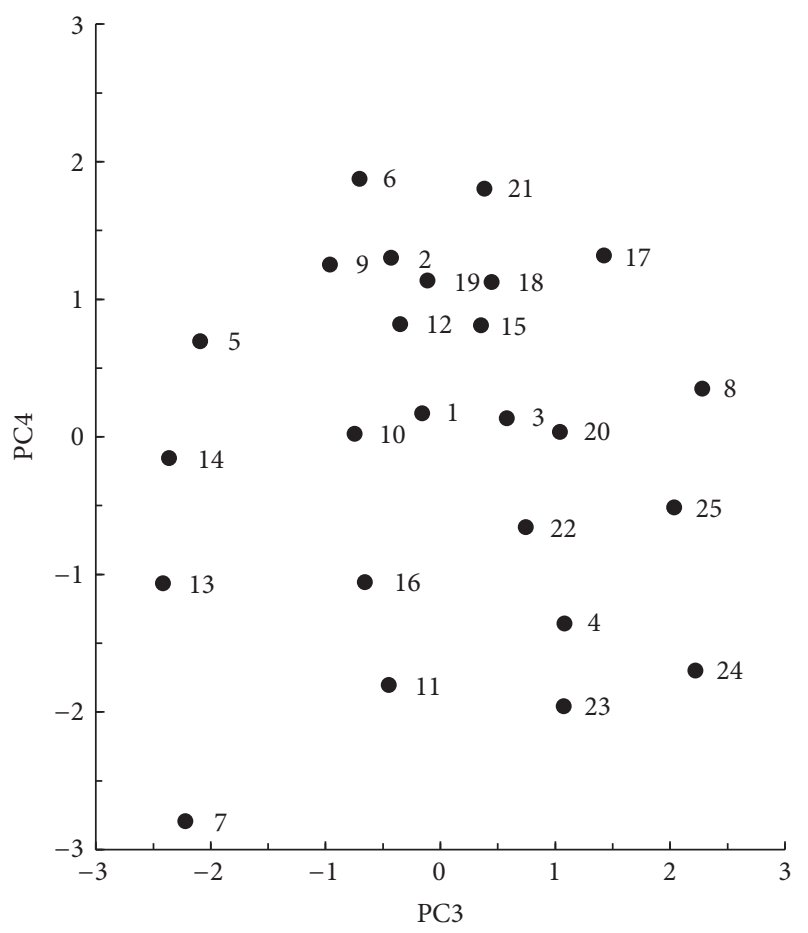

FIgURE 4: The score plot of samples in 3 abd 4 principal components (PC3 and PC4).

thus the method which combines instrumental data with principle component analysis could be an innovational and rapid assessment strategy for them.

\section{Disclosure}

This article does not contain any studies with human participants or animals performed by any of the authors.

\section{Competing Interests}

All authors declare no conflict of interests.

\section{Acknowledgments}

This research was supported by The Key Projects in the National Science \& Technology Pillar Program during the twelfth five-year plan period (Grant no. 2014BAD04B03), National Natural Science Foundation of China (Grants nos. 31301504 and 31571879), and Doctoral Program of Higher Education Research Fund (for new teachers, Grant no. 20130093120011).

\section{References}

[1] Y. Zhao and J. Ming, "Application of glycerol monostearate in flour products," Science and Technology of Food Industry, vol. 6, article 119, 2011.

[2] S.-D. Yu, W.-N. Huang, Q.-B. Zou, and M. Tilley, "Effects of technological factors and yeast fermentation on acrylamide content in fried twisted dough-roll," Journal of Food Science, vol. 29, no. 4, pp. 143-148, 2008.

[3] Y. Yang, X. Y. Song, Z. J. Dong et al., "Optimization of production conditions for fermented frozen fried bread stick," Journal of Food Science, vol. 32, no. 21, pp. 193-197, 2011.

[4] J. Qing-jun, X. Xi-lin, R. Jiao-yan et al., "Study on optimization formula of aluminum-free twisted fritter," Food and Fermentation Industries, no. 4, pp. 72-76, 2012.

[5] L. Li, L. Wang, H. Qian et al., "Study on deep-fried bread stick," Science and Technology of Food Industry, vol. 23, article 086, 2014.

[6] M. Yang, X. Liu, J. Huang et al., "Quality fritters recipe of aluminum-free," Academic Periodical of Farm Products Processing, vol. 6, article 22, 2013.

[7] M. A. Chauvin, C. F. Ross, M. Pitts, E. Kupferman, and B. Swanson, "Relationship between instrumental and sensory determination of apple and pear texture," Journal of Food Quality, vol. 33, no. 2, pp. 181-198, 2010.

[8] F. R. Harker, J. Maindonald, S. H. Murray, F. A. Gunson, I. C. Hallett, and S. B. Walker, "Sensory interpretation of instrumental measurements 1: texture of apple fruit," Postharvest Biology and Technology, vol. 24, no. 3, pp. 225-239, 2002.

[9] K. M. Blaker, A. Plotto, E. A. Baldwin, and J. W. Olmstead, "Correlation between sensory and instrumental measurements of standard and crisp-texture southern highbush blueberries (Vaccinium corymbosum L. interspecific hybrids)," Journal of the Science of Food \& Agriculture, vol. 94, no. 13, pp. 2785-2793, 2014.

[10] P. L. Brookfield, S. Nicoll, F. A. Gunson, F. R. Harker, and M. Wohlers, "Sensory evaluation by small postharvest teams and the relationship with instrumental measurements of apple texture," Postharvest Biology \& Technology, vol. 59, no. 2, pp. 179-186, 2011.

[11] P. Gunness, O. Kravchuk, S. M. Nottingham, B. R. D’Arcy, and M. J. Gidley, "Sensory analysis of individual strawberry fruit and comparison with instrumental analysis," Postharvest Biology \& Technology, vol. 52, no. 2, pp. 164-172, 2009.

[12] C. Primo-Martín, "Cross-linking of wheat starch improves the crispness of deep-fried battered food," Food Hydrocolloids, vol. 28, no. 1, pp. 53-58, 2012.

[13] I. Antonova, P. Mallikarjunan, and S. E. Duncan, "Correlating objective measurements of crispness in breaded fried chicken nuggets with sensory crispness," Journal of Food Science, vol. 68, no. 4, pp. 1308-1315, 2003.

[14] S. Jensen, L. H. Skibsted, U. Kidmose, and A. K. Thybo, "Addition of cassava flours in bread-making: sensory and textural evaluation," LWT_Food Science and Technology, vol. 60, no. 1, pp. 292-299, 2015.

[15] M. Florek, A. Junkuszew, W. Bojar et al., "Effect of vacuum ageing on instrumental and sensory textural properties of meat from uhruska lambs," Annals of Animal Science, vol. 16, no. 2, pp. 601-609, 2016.

[16] D. Wu, D.-W. Sun, and Y. He, "Novel non-invasive distribution measurement of texture profile analysis (TPA) in salmon fillet by using visible and near infrared hyperspectral imaging," Food Chemistry, vol. 145, no. 4, pp. 417-426, 2014.

[17] L.-Z. Cheong, C.-P. Tan, K. Long, N. A. Idris, M. S. A. Yusoff, and O.-M. Lai, "Baking performance of palm diacylglycerol bakery fats and sensory evaluation of baked products," European Journal of Lipid Science and Technology, vol. 113, no. 2, pp. 253-261, 2011. 

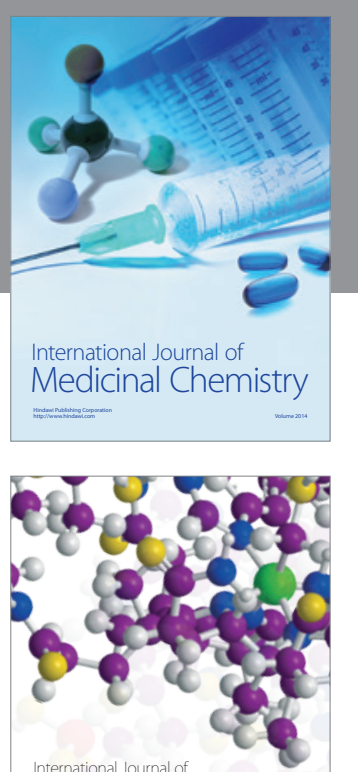

Carbohydrate Chemistry

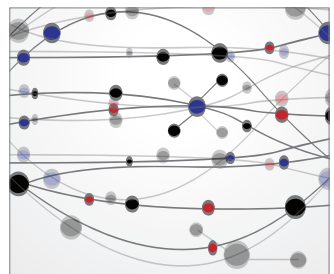

The Scientific World Journal
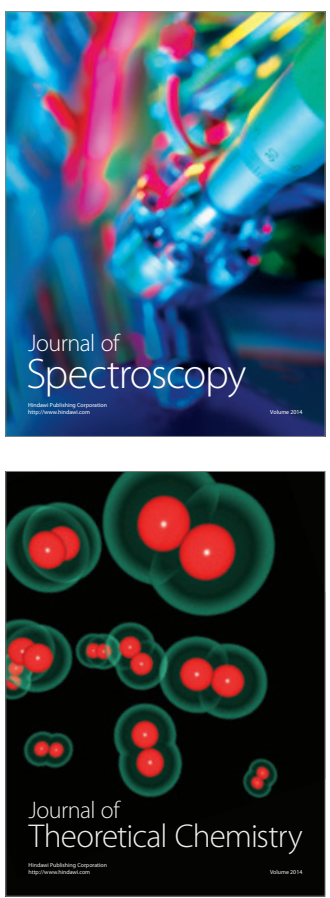
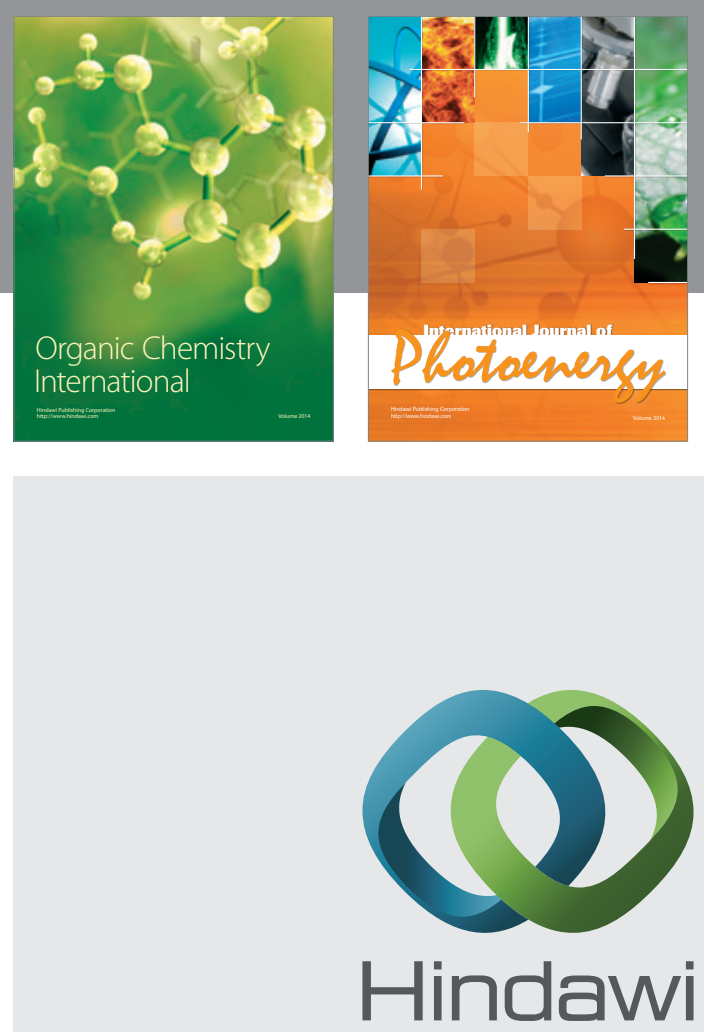

Submit your manuscripts at

http://www.hindawi.com

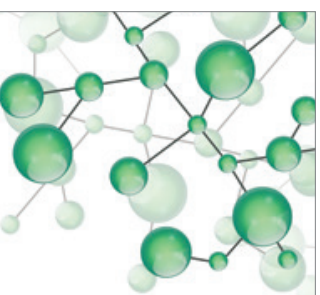

International Journal of

Inorganic Chemistry

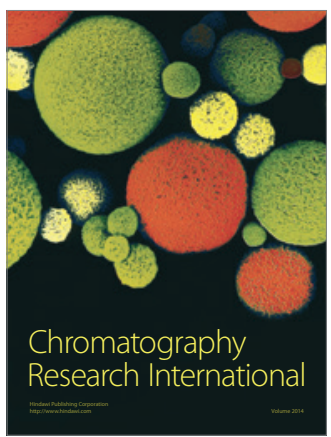

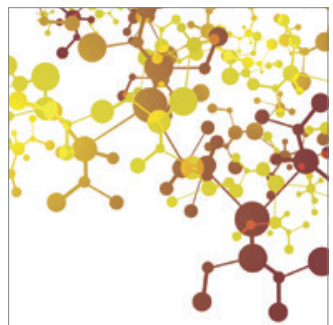

Applied Chemistry
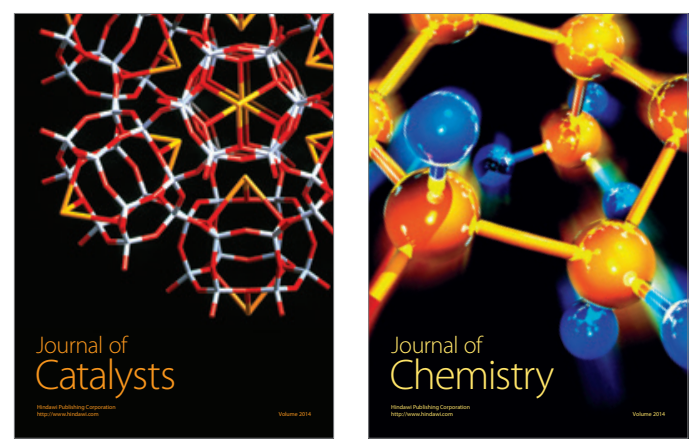
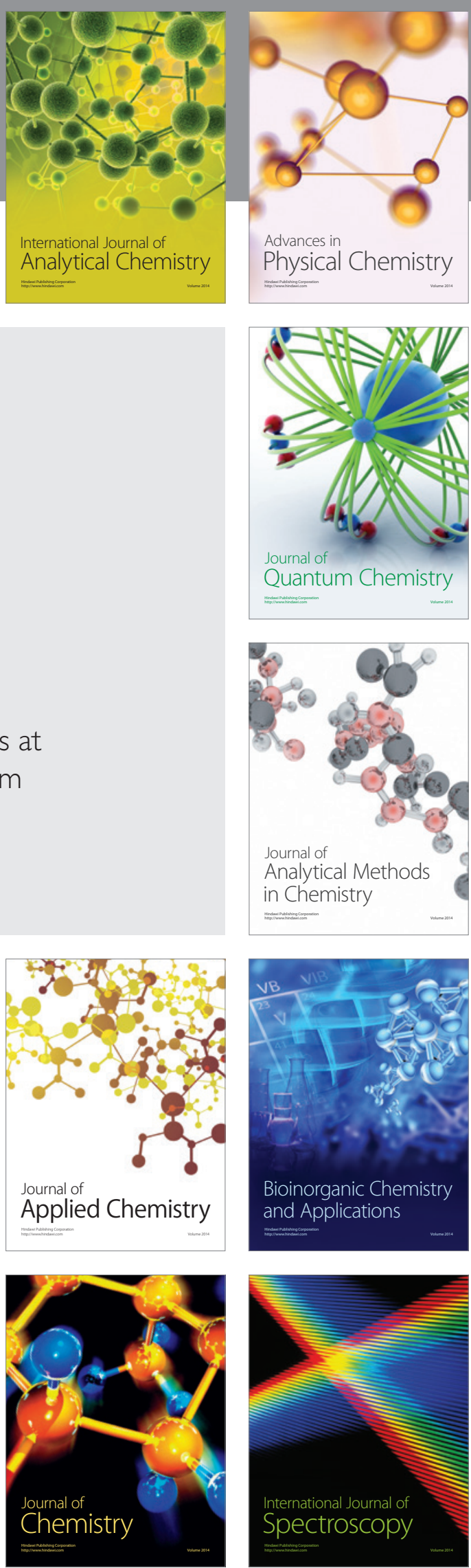